\title{
Some Generalized Results of Fixed Points in Cone b-Metric Spaces
}

\author{
RENy GeORge AND BRIAN FisheR
}

\begin{abstract}
A generalised common fixed point theorem of Tasković type for three mappings $f: X \rightarrow X$ and $S, T: X^{k} \rightarrow X$ in a cone b-metric space is proved. Our result generalises many well-known results.
\end{abstract}

\section{INTRODUCTION}

Fixed point theory has got wide applications in different branches of mathematics. In 1922, the work of S. Banach known as the Banach Contraction Principle, laid the foundation for studies of fixed point theory. Since then, many mathematicians have extended and generalised the work of Banach in different directions. In 1976 Tasković [22] proved a localization theorem on a cartesian product of metric spaces as a solution of Kuratowski's problem of 1932, see: Brown [24]. Later many such results were proved by many authors including Presic [1], Ciric and Presic [2] etc. In [21], Bakhtin introduced the concept of a $b$-metric space as a generalisation of a metric space and proved the contraction mapping theorem in a $b$-metric space. Since then, several papers have dealt with fixed point theory or variational principle for single-valued and multivalued mappings in $b$-metric spaces. Huang and Zang [3], generalising the notion of a metric space by replacing the set of real numbers by ordered normed spaces, defined a cone metric space and proved some fixed point theorems of contractive mappings defined on these spaces. Rezapour and Hamlbarani [4], omitting the assumption of normality, obtained generalisations of results of [3]. In [5], Di Bari and Vetro obtained results on points of coincidence and common fixed points in non-normal cone metric spaces. Further results on fixed point theorems in such spaces were obtained by several authors, see [5-15]. In [20], Hussein and Shah introduced cone $b$-metric spaces as a generalisation of $b$-metric spaces and cone metric spaces, established some topological properties in such spaces and improved

2010 Mathematics Subject Classification. 47H10.

Key words and phrases. Coincidence and common fixed points, cone b-metric space, weakly compatible mappings. 
some results of KKM mappings in the setting of a cone $b$-metric space. Cone $b$-metric spaces play a useful role in fixed point theory. In fact there exist mappings with common fixed points which are contraction mappings in a cone $b$-metric space but are not contraction mappings when defined in a cone metric space. Example 1 of this paper illustrates this fact. The purpose of this paper is to prove a common fixed point theorem of Tasković type for three mappings in non-normal cone $b$-metric spaces, which will extend and generalise recent results of Huang and Xu [19], George and Khan[16], Rao et al. [18], George et al. [17] and also many existing results in metric spaces, $b$-metric spaces and cone $b$-metric spaces.

\section{Preliminaries}

Let $E$ be a real Banach space and $P$ a subset of $E$. Then $P$ is called a cone if

(i) $P$ is closed, non-empty and satisfies $P \neq\{0\}$,

(ii) $a x+b y \in P$ for all $x, y \in P$ and non-negative real numbers $a, b$,

(iii) $x \in P$ and $-x \in P \Rightarrow x=0$, i.e. $P \cap(-P)=0$.

Given a cone $P \subset E$, we define a partial ordering $\preceq$ with respect to $P$ by $x \preceq y$ if and only if $y-x \in P$. We shall write $x<y$ if $x \preceq y$ and $x \neq y$, and $x \ll y$ if $y-x \in \operatorname{int} P$, where int $P$ denotes the interior of $P$. The cone $P$ is called normal if there is a number $K>0$ such that for all $x, y \in E$, $0 \preceq x \preceq y$ implies $\|x\| \preceq K\|y\|$.

Definition $1([3])$. Let $X$ be a non empty set. Suppose that the mapping $d: X \times X \rightarrow E$ satisfies:

$\left(d_{1}\right) \theta \preceq d(x, y)$ for all $x, y \in X$ and $d(x, y)=\theta$ if and only if $x=y$,

$\left(d_{2}\right) d(x, y)=d(y, x)$ for all $x, y \in X$,

$\left(d_{3}\right) d(x, y) \preceq d(x, z)+d(z, y)$ for all $x, y, z \in X$.

Then $d$ is called a cone metric on $X$ and $(X, d)$ is called a cone metric space.

Definition 2 ([3]). Let $X$ be a non empty set and $s \geq 1$ be a given real number. Suppose that the mapping $d: X \times X \rightarrow E$ satisfies:

$\left(d_{1}\right) \theta \preceq d(x, y)$ for all $x, y \in X$ and $d(x, y)=\theta$ if and only if $x=y$,

$\left(d_{2}\right) d(x, y)=d(y, x)$ for all $x, y \in X$,

$\left(d_{3}\right) d(x, y) \preceq s .[d(x, z)+d(z, y)]$ for all $x, y, z \in X$.

Then, $d$ is called a cone $b$-metric on $X$ and $(X, d)$ is called a cone $b$-metric space.

Definition $3([3])$. Let $(X, d)$ be a cone $b$-metric space. The sequence $\left\{x_{n}\right\}$ in $X$ is said to be: 
(a) a convergent sequence if for every $c \in E$ with $0 \ll c$, there is $n_{0} \in N$ such that for all $n \geq n_{0}, d\left(x_{n}, x\right) \ll c$ for some $x \in X$. We denote this by $\lim _{n \rightarrow \infty} x_{n}=x$,

(b) a Cauchy sequence if for all $c \in E$ with $0 \ll c$, there is $n_{0} \in N$ such that $d\left(x_{m}, x_{n}\right) \ll c$, for all $m, n \geq n_{0}$,

(c) a cone $b$-metric space $(X, d)$ is said to be complete if every Cauchy sequence in $X$ is convergent in $X$.

(d) a self-map $T$ on $X$ is said to be continuous if $\lim _{n \rightarrow \infty} x_{n}=x$ implies that $\lim _{n \rightarrow \infty} T\left(x_{n}\right)=T(x)$, for every sequence $\left\{x_{n}\right\}$ in $X$.

Definition $4([16])$. Let $(X, d)$ be a metric space, $k$ a positive integer, $T: X^{k} \rightarrow X$ and $f: X \rightarrow X$ be mappings.

(a) An element $x \in X$ is said to be a coincidence point of $f$ and $T$ if and only if $f(x)=T(x, x, \ldots, x)$. If $x=f(x)=T(x, x, \ldots, x)$, then we say that $x$ is a common fixed point of $f$ and $T$. If $w=f(x)=$ $T(x, x, \ldots, x)$, then $w$ is called a point of coincidence of $f$ and $T$.

(b) Mappings $f$ and $T$ are said to be commuting if and only if $f(T(x, x, \ldots, x))=T(f x, f x, \ldots, f x)$ for all $x \in X$.

(c) Mappings $f$ and $T$ are said to be weakly compatible if and only if they commute at their coincidence points.

Remark 1. For $k=1$, the above definitions reduce to the usual definition of commuting and weakly compatible mappings in a metric space.

The set of coincidence points of $f$ and $T$ is denoted by $C(f, T)$.

\section{MAIN RESUlts}

In the following, we consider a function $\phi: E^{k} \rightarrow E$ such that

(a) $\phi$ is an increasing function, i.e., $x_{1} \preceq y_{1}, x_{2} \preceq y_{2}, \ldots, x_{k} \preceq y_{k}$ implies $\phi\left(x_{1}, x_{2}, \ldots, x_{k}\right) \preceq \phi\left(y_{1}, y_{2}, \ldots, y_{k}\right)$,

(b) $\phi(t, t, t, \ldots) \preceq t$, for all $t \in X$.

Now, we present our main results as follows:

Theorem 1. Let $(X, d)$ be a complete cone b-metric space with coefficient $s \geq 1$ and solid cone $P$ contained in a real Banach space $E$. For any positive integer $k$, let $S, T: X^{k} \rightarrow X$ and $f: X \rightarrow X$ be mappings satisfying the following conditions:

$$
\begin{aligned}
& \qquad S\left(X^{k}\right) \bigcup T\left(X^{k}\right) \subseteq f(X) \\
& \max _{\substack{\beta=S \vee T \\
\gamma=S \vee T}}\left\{d\left(\beta\left(x_{1}, x_{2}, \ldots, x_{k}\right), \gamma\left(x_{2}, x_{3}, \ldots, x_{k+1}\right)\right)\right\} \\
& \preceq \lambda \phi\left(d\left(f x_{1}, f x_{2}\right), d\left(f x_{2}, f x_{3}\right), \ldots,\left(f x_{k}, f x_{k+1}\right)\right) \\
& \quad \text { for all } x_{1}, x_{2}, \ldots, x_{k+1} \in X, \quad s^{k} \lambda \in(0,1),
\end{aligned}
$$




$$
f(X) \text { is complete, }
$$

there exist elements $x_{1}, x_{2}, \ldots, x_{k}, x_{k+1}$ in $X$ and $R$ in $E$ such that $f x_{k+1}=T\left(x_{1}, x_{2}, \ldots, x_{k}\right), \quad 0 \ll R$ and $R$ is the upper bound of the set $\left\{\frac{d\left(f x_{1}, f x_{2}\right)}{\theta}, \frac{d\left(f x_{2}, f x_{3}\right)}{\theta^{2}}, \ldots, \frac{d\left(f x_{k}, f x_{k+1}\right)}{\theta^{k}}\right\}$, where $\theta=\lambda^{\frac{1}{k}}$.

Then $f$ and $T$ have a coincidence point, i.e. $C(f, T) \neq \emptyset$.

Proof. By (3.1)and (3.4) we define a sequence $\left\langle y_{n}>\right.$ in $f(X)$ by $y_{n}=f x_{n}$ for $n=1,2, \ldots, k, y_{k+2 n-1}=f\left(x_{k+2 n-1}\right)=S\left(x_{2 n-1}, x_{2 n}, \ldots, x_{2 n+k-2}\right)$ and $y_{k+2 n}=f\left(x_{k+2 n}\right)=T\left(x_{2 n}, x_{2 n+1}, \ldots, x_{2 n+k-1}\right)$ for $n=1,2, \ldots$

Let $\alpha_{n}=d\left(y_{n}, y_{n+1}\right)$. Then by the method of mathematical induction, we will now prove that

$$
\alpha_{n} \preceq R \cdot \theta^{n}
$$

for all $n$. Clearly by the definition of $R,(3.5)$ is true for $n=1,2, \ldots, k$. Let the $k$ inequalities $\alpha_{n} \preceq R \theta^{n}, \alpha_{n+1} \leq R \theta^{n+1}, \ldots, \alpha_{n+k-1} \preceq R \theta^{n+k-1}$ be the induction hypothesis. Then for any odd $n$ we have

$$
\begin{aligned}
\alpha_{k+n} & =d\left(y_{k+n}, y_{k+n+1}\right) \\
& =d\left(S\left(x_{n}, x_{n+1}, \ldots, x_{n+k-1}\right), T\left(x_{n+1}, x_{n+2}, \ldots, x_{n+k}\right)\right) \\
& \preceq \lambda \phi\left(d\left(f x_{n}, f x_{n+1}\right), d\left(f x_{n+1}, f x_{n+2}\right), \ldots, d\left(f x_{n+k-1}, f x_{n+k}\right)\right) \\
& =\lambda \phi\left(\alpha_{n}, \alpha_{n+1}, \ldots, \alpha_{n+k-1}\right) \\
& \preceq \lambda \phi\left(R \theta^{n}, R \theta^{n+1}, \ldots, R \theta^{n+k-1}\right) \\
& \preceq \lambda \phi\left(R \theta^{n}, R \theta^{n}, \ldots, R \theta^{n}\right) \preceq \lambda R \theta^{n}=R \theta^{n+k} .
\end{aligned}
$$

Further

$$
\begin{aligned}
\alpha_{k+n+1} & =d\left(y_{k+n+1}, y_{k+n+2}\right) \\
& =d\left(T\left(x_{n+1}, x_{n+2}, \ldots, x_{n+k}\right), T\left(x_{n+2}, x_{n+3}, \ldots, x_{n+k+1}\right)\right) \\
& \preceq \lambda \phi\left(d\left(f x_{n+1}, f x_{n+2}\right), d\left(f x_{n+2}, f x_{n+3}\right), \ldots, d\left(f x_{n+k}, f x_{n+k+1}\right)\right) \\
& =\lambda \phi\left(\alpha_{n+1}, \alpha_{n+2}, \ldots, \alpha_{n+k}\right) \\
& \preceq \lambda \phi\left(R \theta^{n+1}, R \theta^{n+2}, \ldots, R \theta^{n+k}\right) \\
& \preceq \lambda \phi\left(R \theta^{n+1}, R \theta^{n+1}, \ldots, R \theta^{n+1}\right) \preceq \lambda R \theta^{n+1}=R \theta^{k+n+1} .
\end{aligned}
$$

Thus inductive proof of (3.5) is complete. 
Now for all $n, p \in N$, we have

$$
\begin{aligned}
d\left(y_{n}, y_{n+p}\right) & \preceq s . d\left(y_{n}, y_{n+1}\right)+s^{2} d\left(y_{n+1}, y_{n+2}\right)+\cdots+s^{p} d\left(y_{n+p-1}, y_{n+p}\right), \\
& \preceq s R \theta^{n}+s^{2} R \theta^{n+1}+\cdots+s^{p} R \theta^{n+p-1} \\
& \preceq s^{p}\left(R \theta^{n}+R \theta^{n+1}+\cdots+R \theta^{n+p-1}\right) \\
& \preceq s^{p} R \theta^{n}\left(1+\theta+\theta^{2}+\cdots\right)=\frac{s^{p} R \theta^{n}}{1-\theta} .
\end{aligned}
$$

Now let $0 \ll c$ be given. Choose $\delta>0$ such that $c+N_{\delta}(0) \subseteq P$, where $N_{\delta}(0)=\{y \in E ;\|y\|<\delta\}$. Also choose a natural number $N_{1}$ such that $\frac{s^{p} R \theta^{n}}{1-\theta} \in N_{\delta}(0)$, for all $n \geq N_{1}$. Then $\frac{s^{p} \cdot R \theta^{n}}{1-\theta} \ll c$ for all $n \geq N_{1}$ and so $d\left(y_{n}, y_{n+p}\right) \preceq \frac{s^{p} . R \theta^{n}}{1-\theta} \ll c$ for all $n \geq N_{1}$.

Hence, the sequence $\left\langle y_{n}\right\rangle$ is a Cauchy sequence in $f(X)$ and since $f(X)$ is complete, there exists $v, u \in X$ such that $\lim _{n \rightarrow \infty} y_{n}=v=f(u)$.

Now choose a natural number $N_{2}$ such that $d\left(y_{n}, y_{n+1}\right) \ll \frac{c}{s^{k+1} \lambda(k+1)}$ and $d\left(v, y_{n}\right) \ll \frac{c}{s(k+1)}$ for all $n \geq N_{2}$. Suppose $k$ is even. Then for all $n \geq N_{2}$

$$
\begin{aligned}
& d(f u, S(u, u, \ldots u)) \preceq s\left[d\left(f u, y_{k+2 n-1}\right)+d\left(y_{k+2 n-1}, S(u, u, \ldots, u)\right)\right] \\
= & s\left[d\left(f u, y_{k+2 n-1}\right)+d\left(S\left(x_{2 n-1}, x_{2 n}, \ldots, x_{k+2 n-2}\right), S(u, u, \ldots, u)\right)\right] \\
\preceq & s d\left(f u, y_{k+2 n-1}\right)+s^{2} d\left(S(u, u, \ldots, u), T\left(u, u, \ldots, x_{2 n-1}\right)\right) \\
& +s^{3} \cdot d\left(T\left(u, u, \ldots x_{n}\right), S\left(u, u, \ldots, x_{2 n-1}, x_{2 n}\right)\right) \\
& +\cdots s^{k} \cdot d\left(T\left(u, x_{2 n-1}, \ldots, x_{k+2 n-3}\right), S\left(x_{2 n-1}, x_{2 n+}, \ldots, x_{k+2 n-2}\right)\right. \\
\preceq & s d\left(f u, y_{k+2 n-1}\right)+s^{2} \lambda \phi\left\{d(f u, f u), d(f u, f u), \ldots, d\left(f u, f x_{2 n-1}\right)\right\} \\
& +s^{3} \lambda \phi\left\{d(f u, f u), d(f u, f u), \ldots, d\left(f u, f x_{2 n-1}\right), d\left(f x_{2 n-1}, f x_{2 n}\right)\right\}+\cdots \\
& +s^{k} \cdot \lambda \phi\left\{d\left(f u, f x_{2 n-1}\right), d\left(f x_{2 n-1}, f x_{2 n}\right), \ldots, d\left(f x_{k+2 n-3}, f x_{k+2 n-2}\right)\right\} \\
= & s d\left(f u, y_{n+k}\right)+s^{2} \lambda \phi\left(0,0, \ldots, d\left(f u, f x_{2 n-1}\right)\right) \\
& +s^{3} \lambda \phi\left(0,0, \ldots, d\left(f u, f x_{2 n-1}\right), d\left(f x_{2 n-1}, f x_{2 n}\right)\right)+\cdots \\
& +s^{k+1} \lambda \phi\left(d\left(f u, f x_{2 n-1}\right), d\left(f x_{2 n-1}, f x_{2 n}\right), \ldots, d\left(f x_{k+2 n-3}, f x_{k+2 n-2}\right)\right) \\
\ll & \frac{c}{s(k+1)}+s^{2} \lambda \phi\left(\frac{c}{s^{k+1} \lambda(k+1)}, \frac{c}{s^{k+1} \lambda(k+1)}, \ldots, \frac{c}{s^{k+1} \lambda(k+1)}\right) \\
& +s^{3} \lambda \phi\left(\frac{c}{s^{k+1} \lambda(k+1)}, \frac{c}{s^{k+1} \lambda(k+1)}, \ldots, \frac{c}{s^{k+1} \lambda(k+1)}\right) \\
& +\cdots+s^{k+1} \lambda \phi\left(\frac{c}{s^{k+1} \lambda(k+1)}, \frac{c}{s^{k+1} \lambda(k+1)}, \ldots, \frac{c}{s^{k+1} \lambda(k+1)}\right) \\
\ll & \frac{c}{k+1}+\lambda \frac{c}{\lambda(k+1)}+\cdots+\lambda \frac{c}{\lambda(k+1)}=c .
\end{aligned}
$$


If $k$ is odd, then for all $n \geq N_{2}$

$$
\begin{aligned}
& d(f u, S(u, u, \ldots, u)) \preceq s \cdot\left[d\left(f u, y_{k+2 n-1}\right)+d\left(y_{k+2 n-1}, S(u, u, \ldots, u)\right)\right] \\
= & s\left[d\left(f u, y_{k+2 n-1}\right)+d\left(S\left(x_{2 n-1}, x_{2 n}, \ldots, x_{k+2 n-2}\right), S(u, u, \ldots, u)\right)\right] \\
\preceq & s d\left(f u, y_{k+2 n-1}\right)+s^{2} d\left(S(u, u, \ldots, u), T\left(u, u, \ldots, x_{2 n-1}\right)\right) \\
& +s^{3} d\left(T\left(u, u, \ldots, x_{n}\right), S\left(u, u, \ldots, x_{2 n-1}, x_{2 n}\right)\right)+\ldots \\
& +s^{k-1} d\left(T\left(u, u, x_{2 n-1}, \ldots, x_{k+2 n-4}\right), S\left(u, x_{2 n-1}, x_{2 n}, \ldots, x_{k+2 n-3}\right)\right. \\
& +s^{k} d\left(S\left(u, x_{2 n-1}, \ldots, x_{k+2 n-3}\right), S\left(x_{2 n-1}, x_{2 n}, \ldots, x_{k+2 n-2}\right)\right.
\end{aligned}
$$

Proceeding as above we get $d(f u, S(u, u, \ldots u)) \ll c$.

Thus, $d(f u, S(u, u, \ldots, u)) \ll \frac{c}{m}$ for all $m \geq 1$.

So, $\frac{c}{m}-d(f u, S(u, u, \ldots, u)) \in P$ for all $m \geq 1$. Since $\frac{c}{m} \rightarrow 0$ as $m \rightarrow \infty$ and $P$ is closed, $-d(f u, S(u, u, \ldots, u)) \in P$, but $P \cap(-P)=/ 0 /$. Therefore, $d(f u, S(u, u, \ldots, u))=0$. Thus, $f u=S(u, u, u, \ldots, u)$.

From (3.2) we have

$$
\begin{aligned}
d(f u, T(u, u, \ldots, u) & \leq d(f u, S(u, u, \ldots, u))+ \\
& +d(S(u, u, \ldots, u), T(u, u, \ldots, u))=0,
\end{aligned}
$$

and so $f u=T(u, u, \ldots, u)$, i.e. $C(f, T, S) \neq \emptyset$.

Theorem 2. Let $(X, d)$ be a complete cone b-metric space with solid cone $P$ contained in a real Banach space $E$. For any positive integer $k$, let $S, T$ : $X^{k} \rightarrow X$ and $f: X \rightarrow X$ be mappings satisfying (3.1), (3.2), (3.3) and let there exist elements $x_{1}, x_{2}, \ldots x_{k}$ in $X$ satisfying (3.4). If the pairs $(f, S)$ and $(f, T)$ are weakly compatible pairs, then $f, S$ and $T$ have a unique common fixed point provided any one of the following hold:

$$
\begin{gathered}
d(S(u, u, \ldots, u), T(v, v, \ldots, v)) \ll d(f u, f v) \\
0<\lambda<\frac{s-1}{s^{k}-1} .
\end{gathered}
$$

Moreover if $x_{1}, x_{2}, \ldots, x_{k}$ are arbitrary points in $X$ and for $n \in N, y_{n+k}=$ $f\left(x_{n+k}\right)=T\left(x_{n}, x_{n+1}, \ldots, x_{n+k-1}\right), n=1,2, \ldots$, then the sequence $\left\langle y_{n}>\right.$ is convergent and $\lim y_{n}=f\left(\lim y_{n}\right)=T\left(\lim y_{n}, \lim y_{n}, \ldots, \lim y_{n}\right)$.

Proof. As proved in Theorem 3.1, there exists $v, u \in X$ such that $\lim _{n \rightarrow \infty} y_{n}=$ $v=f(u)=S(u, u, \ldots, u)=T(u, u, \ldots, u)$. Since the pairs $(f, S)$ and $(f, T)$ are weakly compatible pairs, we have $f f u=f(S(u, u, \ldots u)=S(f u, f u, f u \ldots f u)$ and $f f u=f(T(u, u, \ldots u)=T(f u, f u, f u \ldots f u)$.

Suppose (3.6) hold. Then we have

$$
d(f u, f f u)=d(S(u, u, \ldots, u), T(f u, f u, \ldots, f u)) \ll d(f u, f f u) .
$$


Therefore $f u=f f u$, i.e. $f u=v$ is a common fixed point of $f, S$ and $T$. If (3.7) hold, by (3.2) for any even $k$ we have,

$$
\begin{aligned}
d(f f u, f u)= & d(f T(u, u, \ldots, u), T(u, u, \ldots, u)) \\
= & d(T(f u, f u, f u, \ldots, f u), T(u, u, \ldots, u)) \\
\preceq & s d(T(f u, f u, f u, \ldots, f u), S(f u, f u, \ldots, f u, u)) \\
& +s^{2} . d(S(f u, f u, \ldots, f u, u), T(f u, f u, \ldots, u, u))+\cdots \\
& +s^{k-1} \cdot d(S(f u, u, \ldots, u, u), T(u, u, \ldots, u)) \\
\preceq & s \lambda \phi(d(f f u, f f u), \ldots d(f f u, f f u), d(f f u, f u)) \\
+ & s^{2} \lambda \phi(d(f f u, f f u), \ldots, d(f f u, f u), d(f u, f u))+\cdots \\
& +s^{k-1} \lambda \phi(d(f f u, f u), \ldots, d(f u, f u), d(f u, f u)) \\
= & s \lambda \phi(0,0,0, \ldots, d(f f u, f u))+s^{2} \lambda \phi(0,0, \ldots, 0, d(f f u, f u), 0) \\
& +\cdots s^{k-1} \lambda \phi(d(f f u, f u), 0,0, \ldots, 0) \\
\preceq & \left\{s+s^{2}+\cdots+s^{k-1}\right\} \lambda d(f f u, f u) \\
\preceq & \left\{1+s+s^{2}+\cdots+s^{k+1}\right\} \lambda d(f f u, f u) \\
= & \frac{s^{k}-1}{s-1} \lambda d(f f u, f u) .
\end{aligned}
$$

Similarly for any odd $k$ we have

$$
\begin{aligned}
d(f f u, f u)= & d(f T(u, u, \ldots, u), T(u, u, \ldots, u)) \\
= & d(T(f u, f u, f u, \ldots, f u), T(u, u, \ldots, u)) \\
\preceq & s d(T(f u, f u, f u, \ldots, f u), S(f u, f u, \ldots, f u, u)) \\
& +s^{2} d(S(f u, f u, \ldots, f u, u), T(f u, f u, \ldots, u, u))+\cdots \\
& +s^{k-1} . d(T(f u, u, \ldots, u, u), T(u, u, \ldots, u)) \\
\preceq & \frac{s^{k}-1}{s-1} \lambda d(f f u, f u) .
\end{aligned}
$$

Thus for any $k$ By lemma (1.10) we get $d(f f u, f u)=0$ and so $f f u=f u$. Thus we have,

$$
f u=f f u=S(f u, f u, \ldots, u))=T(f u, f u, \ldots, f u),
$$

i.e.,

$$
v=f v=S(v, v, \ldots, v)=T(v, v, \ldots, v)
$$

and

$$
\begin{aligned}
\lim y_{n}=f\left(\lim y_{n}\right) & =S\left(\lim y_{n}, \lim y_{n}, \ldots, \lim y_{n}\right) \\
& =T\left(\lim y_{n}, \lim y_{n}, \ldots, \lim y_{n}\right) .
\end{aligned}
$$


Now suppose $x, y$ be two fixed points of $f$ and $T$. Then if (3.6) holds

$$
\begin{aligned}
d(x, y) & =d(T(x, x, x, \ldots, x), T(y, y, y, \ldots, y)) \\
& \ll d(f x, f y)=d(x, y)
\end{aligned}
$$

and so $x=y$.

If (3.7) holds then proceeding as before we get

$$
\begin{aligned}
d(x, y) & =d(T(x, x, x, \ldots, x), T(y, y, y, \ldots, y)) \\
& \preceq \frac{s^{k}-1}{s-1} \lambda d(x, y) .
\end{aligned}
$$

By Lemma (1.10) we get $d(x, y)=0$, which implies $x=y$. Hence, the common fixed point is unique.

Following the same lines as in the proof of the above theorems we get the following:

Theorem 3. Let $(X, d)$ be a complete cone b-metric space with solid cone $P$ contained in a real Banach space $E$. For any even positive integer $k$, let $S, T: X^{k} \rightarrow X$ and $f: X \rightarrow X$ be mappings satisfying (3.1), (3.3), (3.4) and

$$
\begin{aligned}
& \max _{\substack{\beta=S \vee T \\
\gamma=S \vee T \\
\beta \neq \gamma}}\left\{d\left(\beta\left(x_{1}, x_{2}, \ldots, x_{k}\right), \gamma\left(x_{2}, x_{3}, \ldots, x_{k+1}\right)\right)\right\} \\
& \preceq \lambda \phi\left(d\left(f x_{1}, f x_{2}\right), d\left(f x_{2}, f x_{3}\right), \ldots,\left(f x_{k}, f x_{k+1}\right)\right) \\
& \quad \text { for all } x_{1}, x_{2}, \ldots, x_{k+1} \in X, \quad \lambda \in(0,1) .
\end{aligned}
$$

If the pairs $(f, S)$ and $(f, T)$ are weakly compatible pairs and if one of (3.6) or (3.7) hold, then $f, S$ and $T$ have a unique common fixed point.

Moreover if $x_{1}, x_{2}, \ldots, x_{k}$ are arbitrary points in $X$ and for $n \in N$

$$
y_{n+k}=f\left(x_{n+k}\right)=T\left(x_{n}, x_{n+1}, \ldots, x_{n+k-1}\right), \quad n=1,2, \ldots,
$$

then the sequence $\left\langle y_{n}\right\rangle$ is convergent and

$$
\begin{aligned}
\lim y_{n}=f\left(\lim y_{n}\right) & =S\left(\lim y_{n}, \lim y_{n}, \ldots, \lim y_{n}\right) \\
& =T\left(\lim y_{n}, \lim y_{n}, \ldots, \lim y_{n}\right) .
\end{aligned}
$$

Taking $S=T$ in theorem 3.2 we have the following:

Theorem 4. Let $(X, d)$ be a complete cone b-metric space with solid cone $P$ contained in a real Banach space $E$. For any positive integer $k$, let $T$ : $X^{k} \rightarrow X$ and $f: X \rightarrow X$ be mappings satisfying (3.1), (3.3), (3.4) and

$$
\begin{aligned}
& \left.d\left(T\left(x_{1}, x_{2}, \ldots, x_{k}\right), T\left(x_{2}, x_{3}, \ldots, x_{k+1}\right)\right)\right\} \\
\preceq & \lambda \phi\left(d\left(f x_{1}, f x_{2}\right), d\left(f x_{2}, f x_{3}\right), \ldots,\left(f x_{k}, f x_{k+1}\right)\right) \\
& \text { for all } x_{1}, x_{2}, \ldots, x_{k+1} \in X, \quad \lambda \in(0,1)
\end{aligned}
$$


If the pairs $(f, S)$ and $(f, T)$ are weakly compatible pairs and if one of (3.6) or (3.7) hold, then $f$ and $T$ have a unique common fixed point.

Moreover if $x_{1}, x_{2}, \ldots, x_{k}$ are arbitrary points in $X$ and for $n \in N$

$$
y_{n+k}=f\left(x_{n+k}\right)=T\left(x_{n}, x_{n+1}, \ldots x_{n+k-1}\right), n=1,2, \ldots,
$$

then the sequence $\left\langle y_{n}\right\rangle$ is convergent and

$$
\lim y_{n}=f\left(\lim y_{n}\right)=T\left(\lim y_{n}, \lim y_{n}, \ldots, \lim y_{n}\right) .
$$

Remark 2. Theorem 3.2 is a proper extension and generalisation of the results of George and Khan[16], George et al [17], Rao et al[18] etc.

Remark 3. Applying the general principle of transpose for cones (see [23]) to theorems 3.2 and 3.3 we get extended and generalised versions of results proved in [1], [2] and [22].

Remark 4. If we take $k=1$ in Theorem 3.4, we get the extended and generalised versions of the result of [19], [3] and [4].

Example 1. Let $E=R^{2}, P=\{(x, y) \in E \backslash x, y \geq 0\}, X=\{1,2,3,4\}$ and $d: X \times X \rightarrow E$ such that

$$
d(x, y)= \begin{cases}\left(|x-y|^{-1},|x-y|^{-1}\right), & \text { if } x \neq y \\ (0,0), & \text { if } x=y\end{cases}
$$

Then, $d$ is a cone $b$-metric on $X$. Indeed, $d(x, y) \leq \frac{6}{5}[d(x, z)+d(z, y)]$. However $d(1,2)=(1,1)>\left(\frac{5}{6}, \frac{5}{6}\right)=d(1,4)+d(4,2)$ and so $d$ is not a cone metric on $X$. Let $T: X^{2} \rightarrow X$ and $f: X \rightarrow X$ be defined as follows:

$$
T(x, y)=\left\{\begin{array}{ll}
4, & \text { if } x=y ; \\
0, & \text { if } x \neq y
\end{array} \quad f(x)=x .\right.
$$

We see that $T$ and $f$ satisfy conditions (3.2)and (3.6) with $k=2, \lambda=\frac{3}{4}$ and $\phi\left(x_{1}, x_{2}\right)=\max \left\{x_{1}, x_{2}\right\}$. However in the cone metric space $(X, d)$ with $E=R^{2}, P=\{(x, y) \in E \backslash x, y \geq 0\}, X=\{1,2,3,4\}$ and $d: X \times X \rightarrow E$ given by $d(x, y)=(|x-y|,|x-y|)$, we see that $T$ and $f$ do not satisfy condition (3.2) for $x=y=4$ and $z=1$.

Example 2. Let $E=R^{2}, P=\{(x, y) \in E \backslash x, y \geq 0\}, X=[0,2]$ and $d: X \times X \rightarrow E$ such that $d(x, y)=\left(|x-y|^{3},|x-y|^{3}\right)$. Then, $d$ is a cone $b$-metric on $X$. Indeed, $d(x, y) \leq 8[d(x, z)+d(z, y)]$. However $d(0,2)=(8,8)>(2,2)=d(0,1)+d(1,2)$ and so $d$ is not a cone metric on 
$X$. Let $T: X^{2} \rightarrow X$ and $f: X \rightarrow X$ be defined as follows:

$$
\begin{aligned}
T(x, y) & =\frac{\left(x^{2}+y^{2}\right)}{8}+\frac{3}{4}, & & \text { if }(x, y) \in[0,1] \times[0,1] \\
T(x, y) & =\frac{(x+y)}{8}+\frac{3}{4}, & & \text { if }(x, y) \in[1,2] \times[1,2] \\
T(x, y) & =\frac{\left(x^{2}+y\right)}{8}+\frac{3}{4}, & & \text { if }(x, y) \in[0,1] \times[1,2] \\
T(x, y) & =\frac{\left(x+y^{2}\right)}{8}+\frac{3}{4}, & & \text { if }(x, y) \in[1,2] \times[0,1] \\
f(x) & =x^{2}, & & \text { if } x \in[0,1] \\
f(x) & =x, & & \text { if } x \in[1,2] .
\end{aligned}
$$

We will prove that $T$ and $f$ satisfies condition (3.2). The method is elaborated in Case 1 and the remaining cases are in brief.

Case 1. $x, y, z \in[0,1]$

$$
\begin{aligned}
& d(T(x, y), T(y, z)) \\
= & \left(|T(x, y)-T(y, z)|^{3},|T(x, y)-T(y, z)|^{3}\right) \\
= & \left(\left|\frac{x^{2}-z^{2}}{8}\right|^{3},\left|\frac{x^{2}-z^{2}}{8}\right|^{3}\right) \\
\leq & \left(8\left(\left|\frac{x^{2}-y^{2}}{8}\right|^{3}+\left|\frac{y^{2}-z^{2}}{8}\right|^{3}\right), 8\left(\left|\frac{x^{2}-y^{2}}{8}\right|^{3}+\left|\frac{y^{2}-z^{2}}{8}\right|^{3}\right)\right) . \\
& \cdot\left(\frac{1}{64}\left(\left|x^{2}-y^{2}\right|^{3}+\left|y^{2}-z^{2}\right|^{3}\right), \frac{1}{64}\left(\left|x^{2}-y^{2}\right|^{3}+\left|y^{2}-z^{2}\right|^{3}\right)\right) \\
= & \frac{1}{32} \frac{1}{2}\left[\left(\left|x^{2}-y^{2}\right|^{3},\left|x^{2}-y^{2}\right|^{3}\right)+\left(\left|y^{2}-z^{2}\right|^{3},\left|y^{2}-z^{2}\right|^{3}\right)\right] \\
\leq & \frac{1}{32} \max \{d(f x, f y), d(f y, f z)\}
\end{aligned}
$$

Case 2. $x, y \in[0,1]$ and $z \in[1,2]$

$$
\begin{aligned}
d(T(x, y), T(y, z)) & =\left(\left|\frac{x^{2}+y^{2}}{8}-\frac{y^{2}+z}{4}\right|^{3},\left|\frac{x^{2}+y^{2}}{4}-\frac{y^{2}+z}{8}\right|^{3}\right) \\
& \leq\left(8\left(\left|\frac{x^{2}-y^{2}}{8}\right|^{3}+\left|\frac{y^{2}-z}{8}\right|^{3}\right), 8\left(\left|\frac{x^{2}-y^{2}}{8}\right|^{3}+\left|\frac{y^{2}-z}{8}\right|^{3}\right)\right) \\
& \leq \frac{1}{32} \max \{d(f x, f y), d(f y, f z)\}
\end{aligned}
$$

Case 3. $x \in[0,1]$ and $y, z \in[1,2]$

$$
\begin{aligned}
d(T(x, y), T(y, z)) & =\left(\left|\frac{x^{2}+y}{8}-\frac{y+z}{4}\right|^{3},\left|\frac{x^{2}+y}{8}-\frac{y+z}{4}\right|^{3}\right) \\
& =\left(\left|\frac{x^{2}-z}{8}\right|^{3},\left|\frac{x^{2}-z}{8}\right|^{3}\right) \\
& \leq\left(8\left(\left|\frac{x^{2}-y}{8}\right|^{3}+\left|\frac{y-z}{8}\right|^{3}\right), 8\left(\left|\frac{x^{2}-y}{8}\right|^{3}+\left|\frac{y-z}{8}\right|^{3}\right)\right) \\
& \leq \frac{1}{32} \max \{d(f x, f y), d(f y, f z)\}
\end{aligned}
$$


Case 4. $x, y, z \in[1,2]$

$$
\begin{aligned}
d(T(x, y), T(y, z)) & =\left(\left|\frac{x+y}{8}-\frac{y+z}{8}\right|^{3},\left|\frac{x+y}{8}-y+z 8\right|^{3}\right) \\
& \leq\left(8\left(\left|\frac{x-y}{8}\right|^{3}+\left|\frac{y-z}{8}\right|^{3}\right), 8\left(\left|\frac{x-y}{8}\right|^{3}+\left|\frac{y-z}{8}\right|^{3}\right)\right) \\
& \leq \frac{1}{32} \max \{(f x, f y), d(f y, f z)\} .
\end{aligned}
$$

Similarly in all other cases $d(T(x, y), T(y, z)) \leq \frac{1}{32} \max \{(f x, f y), d(f y, f z)\}$. Thus, $f$ and $T$ satisfy condition (3.2) with $\phi\left(x_{1}, x_{2}\right)=\max \left\{x_{1}, x_{2}\right\}$. We see that $C(f, T)=1, f$ and $T$ commute at 1 . Finally, 1 is the unique common fixed point of $f$ and $T$.

\section{ACKNOWLEDGEMENT}

The authors are thankful to the learned referee for the valuable comments and suggestions which helped them in bringing this paper in its present form.

\section{REFERENCES}

[1] S.B. Presic, Sur la convergence des suites, Comptes. Rendus. de l'Acad. des Sci. de Paris, 260 (1965), 3828-3830.

[2] Lj.B Ciric, S.B. Presic, On Presic type generalisation of Banach contraction principle, Acta. Math. Univ. Com. LXXVI(2) (2007), 143-147.

[3] L.G. Huang, X. Zhang, Cone metric spaces and fixed point theorems of contractive mappings, J. Math. Anal. Appl. 332(2) (2007), 1468-1476.

[4] S. Rezapour, R. Hamlbarani, Some notes on the paper cone metric spaces and fixed point theorems of contractive mappings, Math. Anal. Appl. 345(2) (2008), 719-724.

[5] C. Di Bari, P. Vetro, $\phi$-pairs and common fixed points in cone metric spaces, Rendiconti del circolo Matematico di Palermo 57(2) (2008), 279-285.

[6] M. Abbas, G. Jungck, Common fixed point results for noncommuting mappings without continuity in cone metric spaces, J. Math. Anal. Appl. 341(1) (2008), 416-420.

[7] M. Abbas, B.E. Rhodades, Fixed and periodic point results in cone metric spaces, Appl. Math. Lett. 22(4) (2009), 511-515.

[8] C. Di Bari, P. Vetro, Weakly $\phi$-pairs and common fixed points in cone metric spaces, Rendiconti del circolo Matematico di Palermo 58(1) (2009), 125-132.

[9] D. Ilic, V. Rakocevic, Common fixed points for maps on cone metric space, Math. Anal. Appl. 341(2) (2008), 876-882.

[10] I. Arandjelovic, Z. Kadelburg, S. Radenovic, Boyd-Wong type common fixed point results in cone metric spaces, Appl. Math. Comput. 217 (2011), 7167-7171.

[11] P. Raja, S.M. Vaezpour, Some extensions of Banch's contraction principle in complete cone metric spaces, Fixed Point Theory Appl., Article ID 768294 (2008), 11 pages.

[12] S. Jankovic, Z. Kadelburg, S. Radenovic, On cone metric spaces: a survey, Nonlin. Anal. 74 (2011), 2591-2601.

[13] S. Simic, A note on Stone's, Baire's, Ky Fan's and Dugundj's theorem in tvs-cone metric spaces, Appl. Math. Lett. 24 (2011), 999-1002. 
[14] P. Vetro, Common fixed points in Cone metric spaces, Rendiconti del circolo Matematico di Palermo, Serie II, 56(3) (2007), 464-468.

[15] Z. Kadelburg, S. Radenovic, V. Rakocevic, A note on the equivalence of some metric and cone fixed point results, Appl. Math. Lett. 24 (2011), 370-374.

[16] Reny George and M.S. Khan, On Presic type extension of Banach contraction principle, Int. J. Math. Anal. (21) (2011), 1019-1024.

[17] Reny George, K.P. Reshma and R. Rajagopalan, A generalised fixed point theorem of Presic type in cone metric spaces and application to Markow process, Fixed Point Theory Appl. 2011, Article ID 2011:85.

[18] K.P.R. Rao, Md. Mushtaq Ali and B. Fisher, Some Presic type generalisation of Banach contraction principle, Math. Moravic. 15(1) (2011), 41-47.

[19] H. Huang and S. Xu, Fixed point theorems of contractive mappings in cone b-metric spaces and applications, Fixed Point Theory Appl. 2012, Article ID 2012:220.

[20] N. Hussain, M.H. Shah, KKM mappings in cone b-metric spaces, Computers and Math. Appl. 62 (2011), 1677-1684.

[21] I.A. Bakhtin, Contraction mapping principle in almost metric spaces, Funct. Anals., Gos. Ped. Inst. Unianowsk, 30 26-37, (1989).

[22] M.R. Tasković, Some results in fixed point theory. Publ. Inst. Math., 20(34) (1976), 231-232.

[23] M.R. Tasković, Principles of transpose in fixed point theory for cone metric spaces, Math. Moravica 15(2) (2011), 55-63.

[24] R.F. Brown, The fixed point property and cartesian product, Amer. Math. Monthly. 89 (1982), 654-678.

\section{Reny George}

1: Department of Mathematics

College of Science

Salmanbin Abdulaziz University

AL-KHARJ

Kingdom of Saudi Arabia

2: Department of Mathematics and Computer Science

St. Thomas College

Bhilai, Chhattisgarh

INDIA

E-mail address: renygeorge02@yahoo.com

\section{Brian Fisher}

Department of Mathematics

UNIVERSITY OF LEICESTER

LEICESTER, LE1 7RH

U.K.

E-mail address: fbr@le.ac.uk 